\title{
Subcutaneous Fat Necrosis of the Newborn: A Case Report
}

\author{
J SULTANA $^{\mathrm{a}}$, S YASMEEN ${ }^{\mathrm{b}}$, NI NAZMEc ${ }^{\mathrm{c}}$, MHM MOSLEM $^{\mathrm{d}}$, ZSM HAQUE $^{\mathrm{e}}$, NN FATEMA $^{\mathrm{f}}$
}

\begin{abstract}
Summary:
We report a case of Subcutaneous Fat Necrosis (SCFN) of a newborn which is an uncommon disorder, occurs during the first year of life, and has been attributed to perinatal stress. SCFN usually has a favorable prognosis, with complete autoresolution of subcutaneous lesions
\end{abstract}

\section{Introduction:}

Subcutaneous Fat Necrosis (SCFN) is an inflammatory disorder of adipose tissue affecting primarily the back, buttocks, arms and thighs, and consists of sharply circumscribed, tender, hard, and erythematousviolaceous subcutaneous nodules and plaques. ${ }^{1}$ SCFN occurs may be due to arrested development in fat metabolism. It is a benign condition with autoresolution of skin lesions but may be complicated by some metabolic abnormalities among which hypercalcemia is the commonest. ${ }^{1}$ Regular follow up is required until 6 months of age. ${ }^{1,2}$ We report a case of Subcutaneous Fat Necrosis (SCFN) of a newborn admitted in $\mathrm{CMH}$ Dhaka.

\section{Case Report:}

A 26-day-old male neonate, born at term to a primigravida mother, was brought to $\mathrm{CMH}$ Dhaka with

a. Dr. Jesmin Sultana, Colonel, Child Specialist \& Neonatologist, Combined Military Hospital, Dhaka

b. Dr. Sabina Yasmeen, Lt Colonel, Child Specialist \& Neonatologist, Combined Military Hospital, Dhaka

c. Dr. Nure Ishrat Nazme, Major, Child Specialist, Combined Military Hospital, Dhaka

d. Dr. Mir Hasan Md. Moslem, Major, Medical Officer, Combined Military Hospital, Dhaka

e. Dr. Zaberul SM Haque, Consultant, Pediatrics, PICU, NICU, Asgar Ali Hospital

f. Dr. Nurun Nahar Fatema, Brigadier General, Paediatric Cardiologist, Head of Dept. of Paediatrics, CMH, Dhaka

Address of Correspondence: Dr. Jesmin Sultana, Colonel, Child Specialist \& Neonatologist, Combined Military Hospital, Dhaka. Email: jesminpaed@yahoo.com

Received: 18 March 2017

Accepted: 5 August 2017 within several weeks or months, but it may also be complicated by serious metabolic alterations such as hypercalcemia.

Keywords: SCFN, newborn, hypercalcemia.

(J Bangladesh Coll Phys Surg 2017; 35: 192-195)

tender, firm, erythematous, indurated plaques localized to the arms, forearms, cheeks, neck and shoulder since $3^{\text {rd }}$ day of his age. The baby was born by emergency cesarean section for fetal distress and less fetal movement and liquor was meconium stained. He developed generalized seizure with poor feeding at $2^{\text {nd }}$ day of his age for which he was treated in CMH Jessore for 7 days. As the plaques were gradually increasing in size, the baby was referred to CMH Dhaka.

On examination, the baby was pink, active, normothermic with normal vital signs and had reddish purple nodular swelling over arms, forearms, cheeks, neck and shoulder between and around scapula (Fig.1). The swellings were mildly tender, non-fluctuant and firm; the joints were normal. After admission, the Hemoglobin level was $10.8 \mathrm{~g} / \mathrm{dL}$, Leukocyte count 10,300/cumm with 38\% Neutrophils, 53\% Lymphocytes

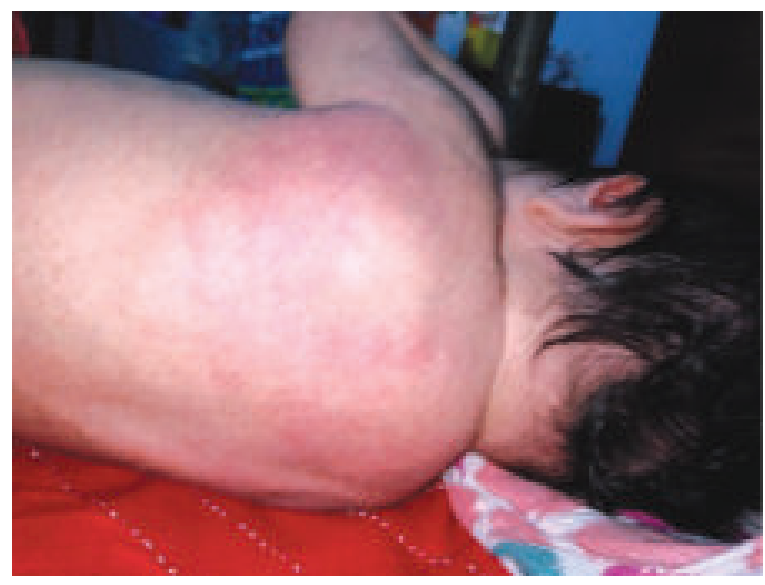

Fig.-1: Erythematous plaques on back of the patient 
and 06\% Eosinophils and Platelet count was 160,000/ cumm. The blood culture and culture of discharge from the swelling was sterile. Blood Urea $10 \mathrm{mg} / \mathrm{dL}$, Serum Creatinine $0.3 \mathrm{mg} / \mathrm{dL}$, Sodium $140 \mathrm{mEq} / \mathrm{L}$, Potassium $4.9 \mathrm{mEq} / \mathrm{L}$, Chloride $98 \mathrm{mEq} / \mathrm{L}$, Calcium $9.6 \mathrm{mg} / \mathrm{dL}$, Magnesium $2.0 \mathrm{mg} / \mathrm{dL}$, Cholesterol $226 \mathrm{mg} / \mathrm{dL}$, Triglyceride 139 mg/dl, HDL 60 mg/dl, LDL 138 mg/dl, Random Blood Sugar $4.5 \mathrm{mmol} / \mathrm{L}, \mathrm{CPK} 28 \mathrm{U} / \mathrm{L}$, Aldolase $5 \mathrm{IU} / \mathrm{L}$ were noted. Chest X-ray, Echocardiography and abdominal Ultrasonogram were normal.

The baby was referred to skin specialist as the lesion gradually extended up to bilateral thigh and buttock. According to his advice serum $\mathrm{C} 3, \mathrm{C} 4, \mathrm{p}-\mathrm{ANCA}, \mathrm{c}-$ ANCA were done which revealed normal. A diagnosis of Subcutaneous Fat Necrosis (SCFN) was made after taking biopsy from the lesion (Fig.-2). The patient was

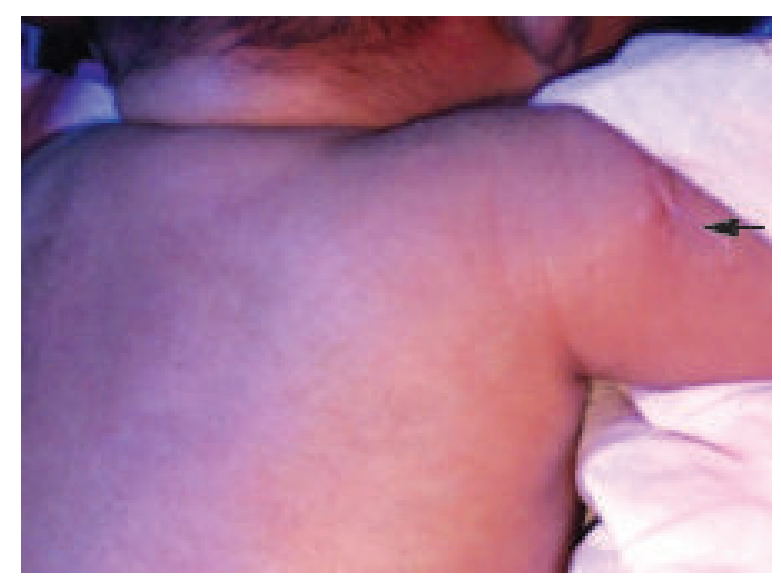

Fig.-2: Biopsy area (arrow) while lesions are almost healed

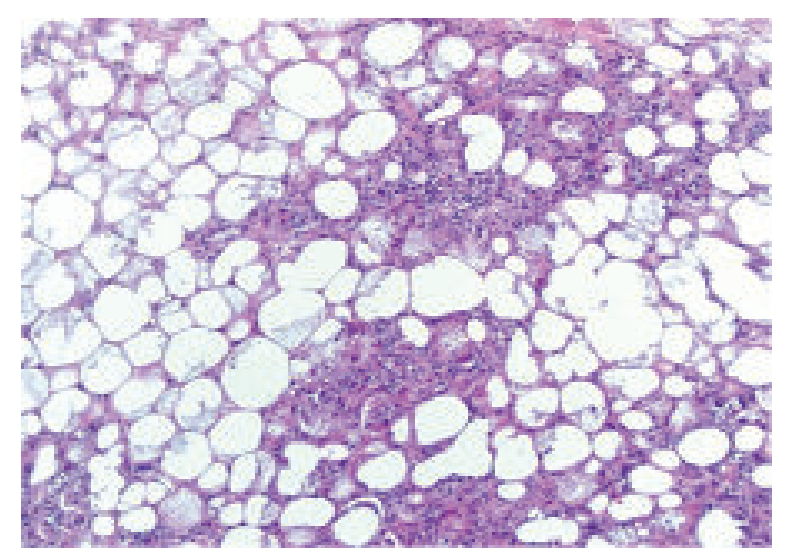

Fig.-3: Histopathological finding of SCFN. Adipocytes in the form of radial spokes and inflammatory reaction with multinucleated giant cells. treated with oral Prednisolone at a dose of $2 \mathrm{mg} / \mathrm{kg} /$ day in 3 divided doses, oral Flucloxacillin and local antibacterial combined with steroid ointment (as a suspected case of myositis due to tender inflamed skin lesion). The patient was discharged 26 days later with serum Calcium level of $10.2 \mathrm{mg} / \mathrm{dL}$ and on treatment with oral Prednisolone and with an advice for routine follow up and regular check up of serum Calcium level upto 6 months of age. The skin lesions absolutely disappeared by the third month without scarrings.

\section{Discussion:}

Subcutaneous fat necrosis (SCFN) is a rare adipose tissue inflammation of the newborn. It is characterized by inflammation and necrosis of subcutaneous fat tissue and typically presenting with firm, well circumscribed, purple-bluish subcutaneous nodules. ${ }^{1}$ Lesions may appear isolated or clustered and are typically located on shoulders, back, buttocks, and face; nodules may evolve into subcutaneous calcifications. It usually appears during the first 6 weeks of life; however, the onset may be delayed up to months after birth. ${ }^{1,2}$

Etiology of this disorder is unknown, but it is linked to multiple neonatal and maternal risk factors. ${ }^{3}$ The maternal risk factors are- preeclampsia, maternal diabetes mellitus, maternal medications (calcium channel blockers, cocaine), smoking or exposure to passive smoking during pregnancy, materno-fetal $\mathrm{Rh}$ incompatibility etc. The neonatal risk factors areumbilical cord prolapsed, meconium aspiration, perinatal asphyxia, therapeutic hypothermia, obstetric trauma, neonatal sepsis and cold exposure etc. ${ }^{3,4}$

The exact pathophysiology of SCFN is also unknown. It has been suggested that an underlying defect in fat composition or metabolism may play an important role in the development of SCFN. In neonates, subcutaneous fat is mostly composed of saturated fatty acids (stearic and palmitic acids) as a result of the inadequately developed enzyme systems involved in fatty acid desaturation. ${ }^{5}$ Neonatal stress may exacerbate this enzymic defect, thus affecting the subcutaneous fat metabolism, leading to an increased fat composition in saturated fatty acids. In addition, with stress, neonatal subcutaneous fat may undergo crystallization due to the high melting point of the saturated fatty acids. This process may lead to adipocyte damage and granulomatous reaction to the resultant fat necrosis. ${ }^{5}$ 
The most frequently recognized possible etiologic factors in a case series of SCFN was birth asphyxia and meconium aspiration syndrome. ${ }^{6}$ Although Obstetric trauma and exposure to cold may act as chief precipitants in SCFN as described by Oza et al., in the case reported herein, it is doubtful that either was contributory. In our case the liquor was meconium stained and there was fetal distress though the baby cried immediately after birth. $^{7}$

The main clinical differential diagnosis of SCFN is sclerema neonatorum ( $\mathrm{SN}$ ). These two entities may be distinguished from each other based on history, physical examination and histological findings. ${ }^{8}$ Diagnosis of SCFN usually requires a deep skin biopsy which shows patchy areas of fat necrosis, surrounded by a granulomatous infiltrate of lymphocytes, macrophages, multinucleated giant cells and abundant histiocytes (Fig.-3). Many of the fat and multi-nucleated giant cells contain needle-shaped clefts that often lie in a radial arrangement in comparisons with SN that shows adipocytes with scattered small clefts and in older lesions with thickened fibrotic septate and calcifications. ${ }^{9,10} \mathrm{~A}$ more benign entity known as cold panniculitis has been described in infants exposed to cold weather, who have had ice applied to the skin, and in children who suck on cold objects ('popsicle panniculitis'). Clinically this condition also resembles SCFN but defers in biopsy finding. ${ }^{11}$ In our patient, the histology was more typical of subcutaneous fat necrosis.

SCFN most often undergoes spontaneous resolution within several weeks but may last as long as 6 months. However, extracutaneous manifestations sometimes occur, and the patient should be evaluated and monitored for hypoglycemia, hypertriglyceridemia, anemia, thrombocytopenia, and (most importantly) hypercalcemia. These metabolic derangements may, in turn, represent a possible risk for serious complications. ${ }^{9,12}$

Hypercalcemia is considered to be an uncommon complication, but to date it is the one most frequently reported. Hypercalcemia appears within 1-6 months after the onset of skin lesions. ${ }^{13}$ Hypercalcemic infants may manifest lethargy, irritability, hypotonia, vomiting, polyuria, polydipsia, dehydration, constipation, and failure to thrive. ${ }^{9}$ Hypercalcemia is caused by increased prostaglandin activity, release of calcium from necrotic fat tissue, and increased secretion of 1, 25dihydroxyvitamin D3 from subcutaneous lesions, leading to an increased intestinal uptake of calcium. If left untreated, moderate to severe hypercalcemia may lead to complications as nephrocalcinosis, nephrolithiasis, renal failure; calcification of falx cerebri, skin, myocardium, and gastric mucosa may also occur. $^{4}$

Thrombocytopenia is usually synchronous with the appearance of subcutaneous nodules, and it is possibly caused by peripheral platelet sequestration into the lesions. ${ }^{9}$ Hypoglycemia is reported in literature as another risk factor linked to SCFN, but rather than being a cause of SCFN it seems to be itself a consequence of hypoxia. Hypertriglyceridemia is caused by mobilization of fatty acids from adipose tissue. ${ }^{5}$

J Dudink presented a case of SCFN with symptomatic hypercalcaemia, who developed calcifications in the liver, the inferior vena cava, and the atrial septum of the heart. ${ }^{14}$ Drut et al. reported seven cases of massive myocardial calcification in the perinatal period in association with SCFN induced hypercalcemia. ${ }^{15} \mathrm{~K}$ Karochristou et al. reported a case of SCFN in a neonate who developed hypocalcaemia instead of hypercalcaemia and a biochemical profile of pseudohypoparathyroidism. Perinatal asphyxia was the only recognized predisposing factor in that patient. ${ }^{16}$ In our case, no metabolic abnormality was discovered by investigations. But the seizure on $2^{\text {nd }}$ day of age may be due to some metabolic error or due to perinatal asphyxia.

Treatment of SCFN consists of analgesia. If hypercalcemia occurs, it may need aggressive treatment. Baseline treatment is hyperhydration, termination of vitamin D substitution and a low calcium diet together with diuretics like furosemide to decrease calcium intake and increase renal calcium output. If these measures do not maintain a normal calcium level prednisone $1-2 \mathrm{mg} /$ $\mathrm{kg} / \mathrm{d}$ is used to reduce the granulomatous inflammatory process and to lower vitamin $\mathrm{D}$ production. Bisphosphonates (e.g., etidronate) may also be employed to treat moderate to severe hypercalcemia when other measures have proven ineffective. ${ }^{13,17}$

Since hypercalcemia may occur after several weeks and last for months, periodical checks of serum calcium are necessary until the age of 6 months of life. Parents should 
be informed of the signs of hypercalcemia like failure to thrive, hypotonia and vomiting. They should be urged to contact their pediatrician, if any should appear. ${ }^{1,2,5}$ Serum Calcium level was monitored regularly in our case upto 6 months of age and it was normal throughout the period.

Conclusions:

Subcutaneous fat necrosis is usually a transient and selflimited condition. However, it may be complicated by a number of metabolic alterations like thrombocytopenia, hypoglycemia, hypercalcemia, and hypertriglyceridemia. It is pivotal to monitor newborns with SCFN to avoid the risk of serious complications, with particular reference to hypercalcemia. Regular monitoring of serum calcium is recommended until the age of 6 months, in infants with history of SCFN.

\section{References:}

1. Gomes MPL, Porro AM, Enokihara MMS, Floriano CM. Subcutaneous fat necrosis of the newborn: clinical manifestations in two cases. An Bras Dermatol 2013; 88(6 Suppl 1): 154-157.

2. Thao Tran J, Sheth AP. Complications of subcutaneous fat necrosis of the newborn: A case report and review of the literature. Pediatr Dermatol 2003;20:257-261.

3. Mahé E, Girszyn N, Hadj-Rabia S, Bodemer C, Hamel-Teillac D, De Prost Y. Subcutaneous fat necrosis of the newborn: a systematic evaluation of risk factors, clinical manifestations, complications and outcome of 16 children. Br J Dermatol. 2007; 156(4):709-15.

4. Szpecht D, Bagnosz-Magnuszewska A, Szymankiewicz M, Gadzinowski J. Subcutaneous fat necrosis in neonates after therapeutic hypothermia - report of two cases. Postepy Dermatol Alergol 2016; 33(2): 152-154.

5. Burden AD, Krafchik BR. Subcutaneous fat necrosis of the newborn: A review of 11 cases. Pediatr Dermatol 1999; 16(5):384-387.
6. Singalavanija S, Limponsanurak W, Wannaprasert T. Subcutaneous Fat Necrosis of the Newborn. J Med Assoc Thai 2007;90(6):1214-20

7. Oza V, Treat J, Cook N, Tetzlaff MT, Yan A. Subcutaneous Fat Necrosis as a Complication of Whole-Body Cooling for Birth Asphyxia. Arch Dermatol 2010; 146(8):882-885.

8. Jardine D, Atherton DJ, Trompeter RS. Sclerema neonatorum and subcutaneous fat necrosis of the newborn in the same infant. Eur J Pediatr. 1990 Dec;150(2):125-6.

9. Tran JT, Sheth A P. Complications of subcutaneous fat necrosis of the newborn: a case report and review of literature. Pediatric Dermatology 2003;20:257-61.

10. Lara LG, Villa AV, Rivas MMO, Capella MS, Prada F, Enseñat MAG. Subcutaneous Fat Necrosis of the Newborn: Report of Five Cases. Pediatrics \& Neonatology 2017; 58(1):85-88.

11. Ter Poorten JC, Hebert AA, Ilkiw R. Cold panniculitis in a neonate. Jam Acad Dermatol1995;33: 383-385.

12. Varan B, Gurakan B, Ozbek N, Emir S. Subcutaneous fat necrosis of the newborn associated with anemia. Pediatric Dermatology 1999;16:381-3.

13. Wiadrowski TP, Marshman G. Subcutaneous fat necrosis of the newborn following hypothermia and complicated by pain and hypercalcaemia. Australas J Dermatol 2001;42:207-210.

14. Dudink J, Walther FJ, Beekman RP. Subcutaneous fat necrosis of the newborn: hypercalcaemia with hepatic and atrial myocardial calcification. Arch Dis Child Fetal Neonatal 2003;88:343-345.

15. Drut R, Drut RM, Greco MA. Massive myocardial calcification in the perinatal period. Pediatr Dev Pathol1998;1:366-74.

16. Karochristou K, Siahanidou T, Kakourou-Tsivitanidou T, Stefanaki K, Mandyla H. Subcutaneous fat necrosis associated with severe hypocalcaemia in a neonate. Journal of Perinatology 2006;26:64-66.

17. Vijayakumar M, Prahlad N, Nammalwar BR, Shanmughasundharam R. Subcutaneous Fat Necrosis with Hypercalcemia. Indian Pediatrics 2005;43:360-363. 